\title{
On the assessment of extremely low breakdown probabilities by an inverse sampling procedure [gaseous insulation]
}

Thyregod, Poul; Vibholm, Svend

Published in:

IEEE Transactions on Electrical Insulation

Link to article, DOI:

$10.1109 / 14.85105$

Publication date:

1991

Document Version

Publisher's PDF, also known as Version of record

Link back to DTU Orbit

Citation (APA):

Thyregod, P., \& Vibholm, S. (1991). On the assessment of extremely low breakdown probabilities by an inverse sampling procedure [gaseous insulation]. IEEE Transactions on Electrical Insulation, 26(3), 367-377.

https://doi.org/10.1109/14.85105

\section{General rights}

Copyright and moral rights for the publications made accessible in the public portal are retained by the authors and/or other copyright owners and it is a condition of accessing publications that users recognise and abide by the legal requirements associated with these rights.

- Users may download and print one copy of any publication from the public portal for the purpose of private study or research.

- You may not further distribute the material or use it for any profit-making activity or commercial gain

- You may freely distribute the URL identifying the publication in the public portal 


\title{
On the Assessment of Extremely Low Breakdown Probabilities by an Inverse Sampling Procedure
}

\author{
Poul Thyregod \\ IMSOR, The Technical University of Denmark, \\ Lyngby, Denmark \\ Svend Vibholm \\ Physics Laboratory II, \\ The Technical University of Denmark, Lyngby, \\ Denmark
}

\begin{abstract}
When assessing extremely low breakdown probabilities for selfrestoring gaseous insulation by automatic equipment, it is often more important to keep the actual number of breakdowns low, rather than to control the total number of voltage applications. In order to achieve this, one might use an inverse sampling procedure that commences the voltage application at a very low level, followed by applications at stepwise increased levels until a breakdown occurs. Following a breakdown, the procedure is restarted at the initial level. The procedure is repeated until a predetermined number of breakdowns have occurred, and the average and standard deviation of the observed first breakdown levels are recorded. In this paper we investigate the statistical properties of such a procedure when the underlying flashover probability function is a double exponential distribution. We describe the relation between the mean of the first breakdown level and the single-shot flashover probability at this mean level, and we show how the sample mean and standard deviation of the first breakdown level may be used to assess this relation in an actual experiment. Finally, we indicate that the results for the double exponential flashover probability function may serve as a good approximation, also when other flashover probability functions are assumed.
\end{abstract}

\section{INTRODUCTION}

$\mathrm{T}$ HE up-and-down test method [1] is widely used for estimation of the $50 \%$ probability flashover voltage $U_{50}$ in self-restoring insulation. When used for the assessment of $U_{50}$, the method is considered to be rather insensitive to the underlying distributional assumptions. If the shape of the underlying flashover probability function is known, the statistical analysis of a series of up-and-down tests may be extended to yield an estimate, not only of $U_{50}$, but also of some parameter $\sigma$, expressing the scale of the flashover probability function. The estimates of $U_{50}$ and $\sigma$ may be combined to yield an estimate of any desired quantile $p$ in the flashover probability function. Thus, assuming a normal distribution, an estimate of the $100 p$ percentile is given by

$$
\hat{U}_{p}=\hat{U}_{50}+\hat{\sigma} z_{p}
$$

with $\hat{U}_{50}$ and $\hat{\sigma}$ denoting the estimates of $U_{50}$ and $\sigma$, respectively, and $z_{p}$ the $100 p$ percentile in the standard 
normal distribution. For a given estimation error on $\hat{U}_{50}$ and $\hat{\sigma}$, the resulting confidence interval for $U_{p}$ tends to be larger, the smaller we choose the value for the desired quantile $p$. It may be shown that the width of the confidence interval is proportional with $z_{p}$. Thus, the further the extrapolation is extended from $U_{50}$, the greater will be the estimation error.

Moreover, the deviation between the estimated value $\hat{U}_{p}$ and the true value $U_{p}$ may be influenced by an improper assumption of flashover probability function. If the standardized $p$-quantile $z_{p}$ corresponding to the normal distribution is used in (1) instead of the p-quantile for the relevant distribution, a systematic estimation error is introduced in the estimate of $U_{p}$. Furthermore, as shown by the authors in [2], the estimates $\hat{U}_{50}$ and $\hat{\sigma}$ will tend to be biased if the breakdown distribution has been wrongly specified.

To overcome these deficiencies, Carrera and Dellera [3] suggested an extended up-and-down method for direct assessment of the quantile corresponding to an arbitrary flashover probability $p$. At each step of the procedure the voltage is applied $m$ times, and the step is followed by an increase $\Delta U$ in voltage if none of these applications resulted in a breakdown, and a decrease $\Delta U$ if at least one breakdown resulted. Under this procedure, the voltage levels for successive steps will tend to fluctuate around a quantile $U_{p}$, with $p$ determined by

$$
p=1-2^{-1 / m}
$$

Thus, for $m=7$, the voltage levels will tend to fluctuate around the $10 \%$ flashover voltage.

Since the extended up-and-down procedure is based upon application of voltages around the desired design value $U_{p}$, with $p$ given by (2) above, the extended up-and-down method is rather insensitive to the underlying distributional assumptions, when used only for assessment of $U_{p}$.

In the testing of gaseous insulants, the insulation may often be considered to be self restoring, unless a breakdown has occurred. In practice, test procedures for such insulants will often be controlled by some automated equipment that only requires manual action when a breakdown has occurred. In such cases considerations concerning the number of breakdowns will often be of greater importance than the toial number of voltage applications. Hence, emphasis is on keeping the number of breakdowns moderate, rather than on limiting the number of total voltage applications.

Under the extended up-and-down procedure the total number of voltage applications is fixed, but the actual number of breakdowns cannot be controlled in advance. In the present paper we shall consider an inverse sampling procedure, i.e. a procedure where the number of breakdowns has been fixed in advance, but the actual number of voltage applications can not be specified in advance.

The idea of applying inverse sampling for the determination of small quantiles dates back to Bartlett [4]. The application of the method under a double exponential flashover probability function was described by the authors in [5]. McLeish and Tosh [6] have discussed the properties of the procedure under an exponential breakdown probability function.

In this paper we shall describe the analysis of first breakdown voltages obtained under the inverse sampling procedure assuming a double exponential flashover probability function. We derive the relation between the flashover probability function and the corresponding distribution of first breakdown voltages under the inverse sampling procedure, and we show how this relation may be utilized to assess the single-shot flashover probability corresponding to the observed average first breakdown voltage.

Since the procedure is based upon voltage applications in the neighborhood of the quantile under investigation, the procedure is found to be insensitive to the underlying distributional assumptions.

\section{THE INVERSE SAMPLING PROCEDURE}

$T^{\mathrm{s}}$ HE procedure consists of $n$ series of successive voltage applications with voltage levels increased stepwise. A series stops when a breakdown has occurred.

The starting voltage $U_{s t}$ and the step voltage $\Delta U$ are chosen to be the same for all series in the test. $U_{s t}$ should be chosen sufficiently small to ensure that no flashover will result from application of the voltage $U_{s t}+\Delta U$.

Each series consists of a number of steps. In step $j$ $(j=1, \ldots, J)$ the voltage $u_{j}=U_{s t}+j \Delta U$ is applied once. (In Section 6 we consider the extended procedure with $m$ voltage applications in each step). If no breakdown results, the procedure continues with step $j+1$. When, at some step $J$, the first breakdown in this series occurs, the series stops, and the procedure restarts with a new series of voltage applications commencing with step 1 , i.e., voltage $u_{1}=U_{s t}+\Delta U$.

The procedure stops when $n$ series have been completed, i.e., following the $n$-th breakdown. 
The test result from each series $i(i=1,2, \ldots, n)$ is the step number $j_{i}$ of the first breakdown, and the corresponding first breakdown voltage $u_{i}^{*}=U_{s t}+j_{i} \Delta U$.

The result of the inverse sampling procedure may be summarized by the average

$$
\bar{u}^{*}=\sum_{i=1}^{n} \frac{u_{i}^{*}}{n}
$$

of the breakdown voltages in the $n$ series, and the estimated variance

$$
s^{* 2}=\sum_{i=1}^{n} \frac{\left(u_{i}^{*}-\bar{u}^{*}\right)^{2}}{(n-1)}
$$

of the breakdown voltages.

The average $\bar{u}^{*}$ represents some quantile $U_{p}$ in the flashover probability function. The corresponding flashover probability $p^{*}$ in general will depend upon the starting value $U_{s t}$, and the step size. In Section 4 we shall investigate this relation, and demonstrate how the flashover probability $p^{*}$ associated with the observed average breakdown voltage may be determined from $\bar{u}^{*}$ and $s^{* 2}$.

\section{THE SAMPLING DISTRIBUTION}

T ET $F(u)$ denote the flashover probability function, i.e. $\bigcup_{F\left(u_{j}\right)}$ gives the probability that a flashover will occur when the voltage $u_{j}=U_{s t}+j \Delta U$ is applied in a single shot. It is well known that $F(u)$ may be interpreted as the relative frequency of flashovers under repeated applications of the voltage $u$.

Consider a single series of the inverse sampling procedure. The probability that the first breakdown occurs at the first step, i.e., when testing at the voltage level $u_{1}$ is

$$
p_{1}=F\left(u_{1}\right)
$$

The probability $p_{2}$ that the first breakdown occurs at the second step is found as the probability of no flashover in the first step, followed by a flashover in the second step

$$
p_{2}=\left\{1-F\left(u_{1}\right)\right\} F\left(u_{2}\right)
$$

Proceeding in this manner one obtains the probability $p_{j}$ that the first flashover occurs at step $j$, i.e., when testing at level $u_{j}(j=1,2, \ldots)$,

$$
p_{j}=\left[\prod_{\nu=1}^{j-1}\left\{1-F\left(u_{\nu}\right)\right\}\right] F\left(u_{j}\right) \quad j=1,2, \ldots
$$

It may be verified that

$$
\sum_{j=1}^{\infty} p_{j}=1
$$

The probabilities given by (7) define a probability distribution on the lattice of voltage levels $u_{j}(j=1,2, \ldots)$. The probabilities give the relative frequencies of first breakdown voltages that would result from a large number of repeated series of the inverse sampling procedure.

The probability distribution (7) has the character of a statistical waiting time distribution associated with the single-shot flashover probability function $F(u)$. In the following we shall use the term 'flashover probability function' to denote the single-shot probability function $F(u)$, and 'first-breakdown distribution' to denote the corresponding waiting time distribution (7) on the lattice of voltage levels $u_{j}(j=1,2, \ldots)$.

The $n$ breakdown voltages $u_{1}^{*}, u_{2}^{*}, \ldots u_{n}^{*}$ constitute a sample of size $n$ from the first-breakdown distribution. The mean and variance of $u_{1}^{*}, u_{2}^{*}, \ldots u_{n}^{*}$ are given by

$$
E\left[u^{*}\right]=\sum_{j=1}^{\infty} u_{j} p_{j}=U_{s t}+\Delta U \sum_{j=1}^{\infty} j p_{j}
$$

and

$$
\begin{aligned}
V\left[u^{*}\right] & =\sum_{j=1}^{\infty} u_{j}^{2} p_{j}-E\left[u^{*}\right]^{2} \\
& =(\Delta U)^{2}\left[\sum_{j=1}^{\infty} j^{2} p_{j}-\left\{\sum_{j=1}^{\infty} j p_{j}\right\}^{2}\right]
\end{aligned}
$$

The breakdown voltages $u_{1}^{*}, u_{2}^{*}, \ldots$ obtained in repeated series of the inverse sampling procedure will fluctuate around a quantile $U_{p}^{*}$ in the flashover probability function where $p^{*}$ is determined by

$$
p^{*}=F\left(E\left[u^{*}\right]\right)
$$

\section{A DOUBLE EXPONENTIAL FLASHOVER PROBABILITY}

\subsection{THE SAMPLE MEAN AND VARIANCE}

$\mathrm{T}^{\mathrm{N}}$ this Section we shall consider the distribution (7) in the case where the flashover probability function is 
given by the double exponential distribution with parameters $\alpha$ and $\beta$, i.e.

$$
F(u)=1-\exp \left\{-\exp \left(\frac{u-\alpha}{\beta}\right)\right\}
$$

It is shown in the Appendix that $p_{j}$ may be determined as the probability associated with the interval $\left[u_{j-1}, u_{j}\right]$ in the approximating first-breakdown distribution $F^{*}$

$$
p_{j}=F^{*}\left(u_{j}\right)-F^{*}\left(u_{j-1}\right)
$$

where the distribution $F^{*}$ is obtained from the flashover probability function $F$ as

$$
F^{*}(u)= \begin{cases}\frac{F(u+\eta \beta)}{F\left(U_{s t}+\eta \beta\right)} & \text { if } u>U_{s t} \\ 0 & \text { if } u \leqslant U_{s t}\end{cases}
$$

with the displacement parameter $\eta$ determined by

$$
\eta=\log \left[\frac{\exp (\Delta U / \beta)}{\exp (\Delta U / \beta)-1}\right]
$$

The approximating first-breakdown distribution $F^{*}$ is a continuous distribution that is obtained from the flashover probability function $F$ by displacing the variate origin by $+\eta \beta$ voltage units. Thus with respect to a graphical illustration, $F$ is displaced to the left. The resulting distribution is then truncated at the voltage $U_{s t}$.

The lattice distribution (13) of the first breakdown voltage is obtained by discretization of the approximating continuous distribution. If the step size is not too large, this discretization will be of minor importance, and we may approximate $E\left[u^{*}\right]$ and $V\left[u^{*}\right]$ by the mean and variance of the approximating distribution (14).

Thus, disregarding the truncation and the discretization, we find that the distribution of the first breakdown voltage under inverse sampling is approximately a double exponential distribution obtained by displacing the original flashover probability function $\eta \beta$ units to the left. We therefore have the following approximation to the mean and variance of $u^{*}$

$$
\begin{aligned}
E\left[u^{*}\right] & \approx \alpha-\beta \gamma-\eta \beta \\
V\left[u^{*}\right] & \approx \frac{\pi^{2}}{6} \beta^{2}
\end{aligned}
$$

with $\gamma \approx 0.5772$ denoting Eulers constant.

\subsection{THE ESTIMATED SINGLE-SHOT FLASHOVER PROBABILITY}

The breakdown voltages $u_{1}^{*}, u_{2}^{*}, \ldots$ obtained in the repeated series of the inverse sampling procedure will fluctuate around the mean value $E\left[u^{*}\right]$. This mean value is the $p^{*}$-quantile in the underlying flashover probability function $p^{*}=F\left(E\left[u^{*}\right]\right)$.

In applications of the inverse sampling procedure it is of interest to design the procedure (i.e. the starting value $U_{s t}$ and the step size $\left.\Delta U\right)$ in such a way that $p^{*}$ will be in the neighborhood of some specified low value, e.g. $p^{*} \simeq 0.01$.

Using the approximation (16) we find

$$
p^{*}=F\left(E\left[u^{*}\right]\right) \approx 1-\exp \{-\exp (-\gamma-\eta)\}
$$

Thus if the starting value has been chosen sufficiently small, then the estimated flashover probability under the inverse sampling procedure, $p^{*}$, depends only on the displacement parameter $\eta$, i.e. on the normalized step size $\Delta U / \beta$.

Figure 1 shows the relation between the normalized step size $\Delta U / \beta$ and the estimated flashover probability $p^{*}$ for different starting values of the inverse sampling procedure. On this occasion, $U_{s t}$ is referred to different quantiles $P_{s t}$ in the flashover probability function, i.e. $P_{s t}=F\left(U_{s t}\right)$. It is seen that the relation (18) yields a satisfactory approximation to the estimated flashover probability. The only exceptions are for obvious reasons the situations when the starting value is very close to (or larger than) the approximate value (16) of the expected first breakdown voltage.

Table 1 shows the relation between the normalized step size $\Delta U / \beta$, the displacement $\eta$, and the approximate value (18) of the estimated flashover probability.

When the step size, $\Delta U / \beta$, is sufficiently small we may expand $\eta$ to yield

$$
\begin{aligned}
\eta & =\frac{\Delta U}{\beta}-\log \left\{\exp \left(\frac{\Delta U}{\beta}\right)-1\right\} \\
& \approx \frac{\Delta U}{\beta}-\log \left(\frac{\Delta U}{\beta}\right) \approx-\log \left(\frac{\Delta U}{\beta}\right)
\end{aligned}
$$

such that we obtain

$$
p^{*} \approx 1-\exp \left\{-\left(\frac{\Delta U}{\beta}\right) \exp (-\gamma)\right\} \approx\left(\frac{\Delta U}{\beta}\right) \exp (-\gamma)
$$

Hence, the estimated flashover probability is approximately proportional to the step size, at least for small step sizes. It is seen from Figure 1 and Table 1 that the approximation is fairly good, even for moderate step sizes, i.e. $\Delta U / \beta<0.1$ 
Table 1.

Values of the normalized step size $\Delta U / \beta$, and the corresponding displacement parameter $\eta$ for inverse sampling from a double exponential flashover probability function (exact values). The table also shows the approximate value (18) of the estimated flashover probability

\begin{tabular}{|c||c|c|c|c|c|c|c|c|c|}
\hline \hline$\Delta U / \beta$ & 0.0001 & 0.0002 & 0.0003 & 0.0004 & 0.0005 & 0.0006 & 0.0007 & 0.0008 & 0.0009 \\
\hline$\eta$ & 9.21 & 8.52 & 8.11 & 7.82 & 7.60 & 7.42 & 7.26 & 7.13 & 7.01 \\
\hline $1000 p^{*}$ & 0.06 & 0.11 & 0.17 & 0.22 & 0.28 & 0.34 & 0.39 & 0.45 & 0.51 \\
\hline \hline$\Delta U / \beta$ & 0.001 & 0.002 & 0.003 & 0.004 & 0.005 & 0.006 & 0.007 & 0.008 & 0.009 \\
\hline$\eta$ & 6.91 & 6.22 & 5.81 & 5.52 & 5.30 & 5.12 & 4.97 & 4.83 & 4.72 \\
\hline $1000 p^{*}$ & 0.56 & 1.12 & 1.68 & 2.24 & 2.80 & 3.35 & 3.91 & 4.46 & 5.02 \\
\hline \hline$\Delta U / \beta$ & 0.01 & 0.02 & 0.03 & 0.04 & 0.05 & 0.06 & 0.07 & 0.08 & 0.09 \\
\hline$\eta$ & 4.61 & 3.92 & 3.52 & 3.24 & 3.02 & 2.84 & 2.69 & 2.57 & 2.45 \\
\hline$\overline{1000 p^{*}}$ & 5.57 & 11.06 & 16.46 & 21.77 & 27.01 & 32.17 & 37.25 & 42.25 & 47.18 \\
\hline \hline$\Delta U / \beta$ & 0.10 & 0.20 & 0.30 & 0.40 & 0.50 & 0.60 & 0.70 & 0.80 & 0.90 \\
\hline$\eta$ & 2.35 & 1.71 & 1.35 & 1.11 & 0.93 & 0.80 & 0.69 & 0.60 & 0.52 \\
\hline $1000 p^{*}$ & 52.03 & 96.77 & 135.43 & 168.98 & 198.22 & 223.79 & 246.22 & 265.95 & 283.37 \\
\hline
\end{tabular}

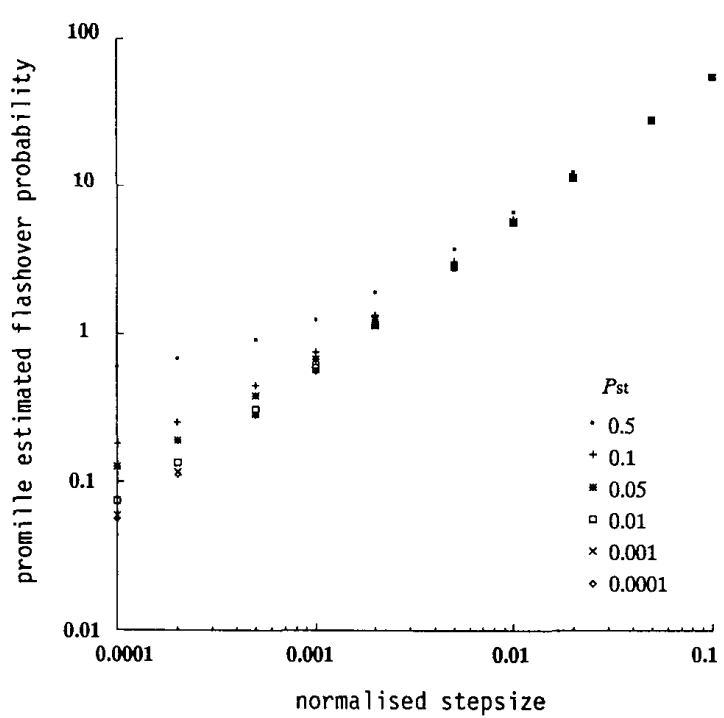

Figure 1.

The estimated flashover probability $p^{*}=F\left(E\left[u^{*}\right]\right)$ as function of the normalized step size $\Delta U / \beta$ for different starting values $U_{s t}=U_{P_{s t}}$ of the inverse sampling procedure. Double exponential flashover probability function. Exact values.

In applications of the inverse sampling procedure the experimenter might insert his prior assessment of the scale parameter $\beta$ into (18) or (20) and choose the step size $\Delta U$ to ensure that the procedure will operate in the neighborhood of the flashover probability of interest.

\subsection{THE FLASHOVER PROBABILITY AND THE SAMPLE VARIANCE}

The scale parameter $\beta$ that enters into (20) is not directly observable. For estimation purposes it is therefore of interest to express the estimated flashover probability $p^{*}$ in terms of an observable quantity.

From (17) we have

$$
\frac{1}{\beta^{2}}=\frac{\pi^{2}}{6 V\left[u^{*}\right]}=\frac{(1.28)^{2}}{V\left[u^{*}\right]}
$$

which inserted into (20) leads to

$$
p^{*} \approx \frac{\Delta U \pi \exp (-\gamma)}{\sqrt{6 V\left[u^{*}\right]}}=\frac{0.72}{\sqrt{V\left[u^{*}\right]} / \Delta U}
$$

Thus, we may express the estimated flashover probability $p^{*}$ as a function of the normalized standard deviation, $\sqrt{V\left[u^{*}\right]} / \Delta U$, of the first breakdown voltage measured in voltage steps.

Figure 2 shows the relation between the estimated flashover probability and the standard deviation of the first-. breakdown voltage for the same combinations of step sizes and starting values as in Figure 1. It is seen that the approximation by $(20)$ is fairly good.

Clearly, when the starting value is close to, or even greater than the approximate value (16) of the expected first breakdown voltage, then the exact variance of the first breakdown voltage will be smaller than the approximate value (17). It is, however, of interest to note that 
the reduction in the variance just suffices to make the approximation (22) valid, even in those cases where the approximation by (18) was found to be inadequate.

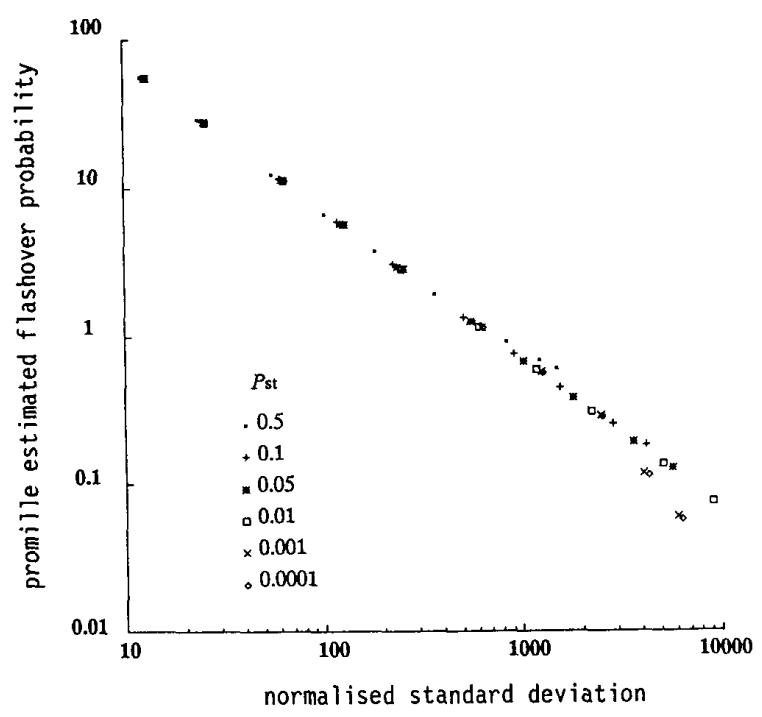

Figure 2.

The estimated flashover probability $p^{*}=F\left(E\left[u^{*}\right]\right)$ as function of the normalized standard deviation $\sqrt{V\left[\boldsymbol{u}^{*}\right]} / \Delta U$ of the first breakdown voltage under the inverse sampling procedure for different starting values $U_{s t}=U_{P_{a t}}$ of the inverse sampling procedure. Exact values.

\section{THE ESTIMATION PROCEDURE}

$T^{N}$ the statistical analysis of test results obtained by the inverse sampling procedure one might use the maximum-likelihood approach to estimate the parameters $\alpha$ and $\beta$. In this presentation we shall, however, present a more accessible approach to the analysis. Our approach will be based upon the method of moments, i.e., we shall utilize the properties of the sample values $\bar{u}^{*}$ and $s^{*^{2}}$ given by (3) and (4) to estimate $\alpha$ and $\beta$, or directly to estimate the flashover probability $p^{*}$ corresponding to $\bar{u}^{*}$.

\subsection{ESTIMATION OF A SPECIFIED QUANTILE}

Let $n$ series of the inverse sampling procedure result in the $n$ first breakdown voltages $u_{1}^{*}, u_{2}^{*}, \ldots u_{n}^{*}$ with the average $\bar{u}^{*}$ and the standard deviation $s^{*}=\sqrt{s^{* 2}}$. We may then utilize (16) and (17) to estimate the parameters $\alpha$ and $\beta$ in the underlying double extreme flashover probability function by

$$
\hat{\beta}=\frac{s^{*} \sqrt{6}}{\pi}
$$

and

$$
\hat{\alpha}=\hat{u}^{*}+\hat{\beta}(\gamma+\hat{\eta})
$$

with

$$
\begin{aligned}
\hat{\eta} & =\log \left[\frac{\exp (\Delta U / \hat{\beta})}{\exp (\Delta U / \hat{\beta})-1}\right] \\
& =\log \left[\frac{\exp \left\{\pi \Delta U /\left(\sqrt{6} s^{*}\right)\right\}}{\exp \left\{\pi \Delta U /\left(\sqrt{6} s^{*}\right)\right\}-1}\right]
\end{aligned}
$$

For a specified value of $p$, we may then estimate the $p$-quantile $U_{p}$ in the flashover probability function by

$$
\hat{U}_{p}=\hat{\alpha}+\hat{\beta} \log (-\log (1-p))
$$

\subsection{THE ESTIMATED FLASHOVER PROBABILITY}

The estimation of a specific quantile $U_{p}$ in the flashover probability function by means of (26) utilizes the assumed form (12) of the flashover probability function to extrapolate $\hat{U}_{p}$ from the observed breakdown voltages $u_{1}^{*}, u_{2}^{*}, \ldots u_{n}^{*}$. Consequently, this estimate will be sensitive to wrong specifications in the form of the flashover probability function. However, when the specified flashover probability $p$ is close to the estimated probability $p^{*}$, the effect of such a wrong specification will be small.

Therefore, it may be of interest to assess directly the single-shot flashover probability $p^{*}$ corresponding to the average first breakdown value $\overline{u^{*}}$ obtained from $n$ series of the inverse sampling procedure.

Inserting the sample standard deviation $s^{*}$ into (22) in place of the theoretical standard deviation $\sqrt{V\left[u^{*}\right]}$, we obtain the estimated flashover probability corresponding to the sample average first breakdown voltage $\bar{u}^{*}$ as

$$
\hat{p}^{*}=\frac{0.72}{s^{*} / \Delta U}
$$

\section{AN EXTENDED INVERSE SAMPLING PROCEDURE}

\footnotetext{
$T_{\text {HE inverse sampling procedure described in Section } 2}$ 1 may be extended to include $m$ voltage applications in
} 
each step, instead of just one application. All $m$ voltage applications in step $j$ will operate at the voltage level $u_{j}=U_{s t}+j \Delta U$. The first-breakdown distribution under this extended procedure is determined by

$$
\begin{aligned}
p_{1} & =1-\left\{1-F\left(u_{1}\right)\right\}^{m} \\
p_{j} & =\left[\prod_{\nu=1}^{j-1}\left\{1-F\left(u_{\nu}\right)\right\}^{m}\right]\left[1-\left\{1-F\left(u_{j}\right)\right\}^{m}\right] \\
j & =2,3, \ldots
\end{aligned}
$$

The double exponential flashover probability function (12) has the property that the probability of no flashover among $m$ voltage application at level $u_{j}$ equals the probability of no flashover in a single voltage application at the displaced level $u_{j}+\beta \log m$ :

$$
\left\{1-F\left(u_{j}\right)\right\}^{m}=1-F\left(u_{j}+\beta \log m\right)
$$

Thus, in this case, the first-breakdown distribution under the extended procedure may be determined from the firstbreakdown distribution (13) corresponding to the simple procedure, by substituting the adjusted starting voltage level $U_{s t}+\beta \log m$ for the starting voltage level $U_{s t}$ in (14). Therefore, assuming a double exponential flashover probability function, the exact first-breakdown distribution under the extended procedure is obtained as

$$
p_{j}=F_{m}^{*}\left(u_{j}\right)-F_{m}^{*}\left(u_{j-1}\right)
$$

where the approximating first-breakdown distribution $F_{m}^{*}$ is given by

$$
F_{m}^{*}(u)= \begin{cases}F\left(u+\frac{\beta[\eta+\log m]}{F\left[U_{s t}+\beta(\eta+\log m)\right]}\right) & u>U_{s t} \\ 0 & u \leqslant U_{s t}\end{cases}
$$

We remark that the first-breakdown distribution corresponding to the extended procedure with step size $\Delta_{m} U$ and $m$ voltage applications at each step is identical to the first-breakdown distribution corresponding to the simple procedure with one voltage application at each step and displacement parameter $\eta_{m}=\eta+\log m$, i.e. with a step size $\Delta U$ satisfying

$$
\begin{aligned}
& \frac{\Delta U}{\beta}-\log \left\{\exp \left(\frac{\Delta U}{\beta}\right)-1\right\} \\
= & \frac{\Delta_{m} U}{\beta}+\log m-\log \left\{\exp \left(\frac{\Delta_{m} U}{\beta}\right)-1\right\}
\end{aligned}
$$

Thus, the properties of the extended procedure are found directly from the properties of the simple procedure by substituting the adjusted displacement parameter $\eta+\log m$ for the displacement parameter $\eta$.
Therefore it follows from Section 4 that

$$
E\left[u^{*}\right] \approx \alpha-\beta(\log m+\eta+\gamma)
$$

and

$$
V\left[u^{*}\right] \approx \frac{\pi^{2}}{6} \beta^{2}
$$

with the corresponding flashover probability

$$
p^{*} \approx \frac{1}{m}\left(\frac{\Delta_{m} U}{\beta}\right) \exp (-\gamma)
$$

The estimates under the extended procedure are

$$
\hat{\beta}=\frac{s^{*} \sqrt{6}}{\pi}
$$

and

$$
\hat{\alpha}=\bar{u}^{*}+\hat{\beta}(\gamma+\log m+\hat{\eta})
$$

with

$$
\hat{\eta}=\log \left[\frac{\exp \left\{\pi \Delta_{m} U /\left(\sqrt{6} s^{*}\right)\right\}}{\exp \left\{\pi \Delta_{m} U /\left(\sqrt{6} s^{*}\right)\right\}-1}\right]
$$

and

$$
\hat{p}_{u^{*}}=\frac{0.72}{m\left(s^{*} / \Delta_{m} U\right)}
$$

It should be noted that only the position of the firstbreakdown distribution is affected by the introduction of multiple voltage applications in each step. The scale parameter in the first-breakdown distribution is the same as in the original flashover probability function.

Figure 3 shows the relation between the estimated flashover probability $p^{*}=F\left(E\left[u^{*}\right]\right)$ and the normalized standard deviation of the first breakdown voltage

$\sqrt{V\left[u^{*}\right]} / \Delta_{m} U$ (in voltage steps) for different choices of $m$. The Figure has been based upon the exact distribution (30). It is seen that the approximative relation (35) yields a good description of the exact relation.

Thus, there is room for some flexibility in the design of the inverse sampling procedure. Given a design value $p^{*}$ of the flashover probability, the designer is free to choose the number $m$ of voltage applications at each step and the step size $\Delta_{m} U$, as long as the ratio $\Delta_{m} U / m$ satisfies (35).

\section{AN EXAMPLE}

A $s$ an illustration of the estimation procedure we con$\mathrm{A}_{\text {sider data from an experimental study previously re- }}$ ported by the authors [5]. The data relate to a study that aimed to estimate the $0.3 \%$ percentile for a specific $\mathrm{SF}_{6}$ system. Thus, according to Table 1, a step size 


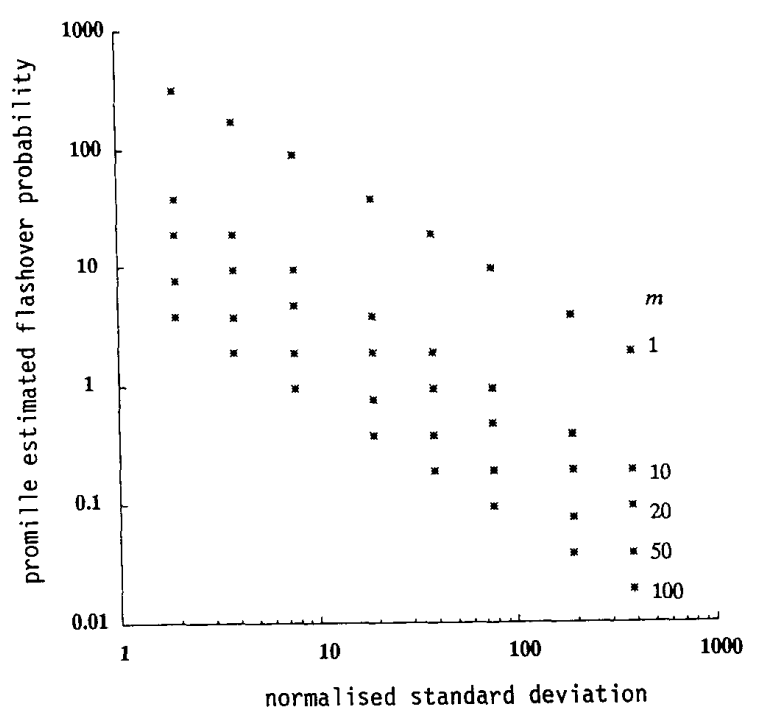

Figure 3.

The estimated flashover probability $p^{*}=F\left(E\left[u^{*}\right]\right)$ and the normalized standard deviation $\sqrt{V\left[u^{*}\right]} / \Delta_{m} U$ for the inverse sampling procedure with $m=1,10,20,50$ and 100 under a double exponential flashover probability function. Normalized step sizes $\Delta_{m} U / \beta=1.0 ; 0.5 ; 0.2 ; 0.1 ; 0.05 ; 0.02 ; 0.01$; $0.005 ; 0.002 ; 0.0002$.

$\Delta U=0.005 \beta$ would be appropriate in a simple inverse sampling procedure. Previous investigations had indicated that a scale parameter $\beta=5 \mathrm{kV}$ might be relevant. Therefore a step size $\Delta U=0.025 \mathrm{kV}$ would be appropriate in a simple inverse sampling procedure. Since it was decided to use an extended inverse sampling procedure with $m=100$ voltage applications per step, the corresponding step size for the extended procedure was found to be $\Delta U=2.5 \mathrm{kV}$. For practical reasons the actual step size was chosen slightly on the safe side, viz. $\Delta U=2.14$ $\mathrm{kV}$.

The starting value was chosen to be $U_{s t}=358 \mathrm{kV}$ which was considered to be sufficiently low to ensure that no flashover would result from voltage applications at the first level $u_{1}=360.14 \mathrm{kV}$.

The experimental results are shown in Table 2. As a check of the experimental procedure, the shot number, at each step level, corresponding to the first breakdown was also recorded. The shot numbers were distributed between 8 and 97 with no systematic pattern.

The average of the first breakdown voltages is found to be $\bar{u}^{*}=432.04 \mathrm{kV}$. The sample variance is $s^{* 2}=$
$(6.40 \mathrm{kV})^{2}$ and the sample standard deviation is $s^{*}=6.40$ $\mathrm{kV}$. The sample standard deviation in terms of voltage steps is $s^{*} / \Delta U=2.99$ steps.

The estimated scale parameter $\beta$, is found as

$$
\hat{\beta}=\frac{s^{*} \sqrt{6}}{\pi}=4.99 \mathrm{kV}
$$

and hence the estimated normalized step size is $\Delta U / \hat{\beta}=$ 0.4289 with the corresponding value of the displacement parameter $\hat{\eta}=1.05$. Thus, we obtain the estimate of the location parameter $\hat{\alpha}=463.16 \mathrm{kV}$. Inserting these values into (26) we finally find the estimated $0.3 \%$ quantile in the flashover probability function as

$U_{0.003}=463.16 \mathrm{kV}-4.99 \mathrm{kV} \log (-\log (0.997))=434.18 \mathrm{kV}$

It was, however, of greater concern to assess directly the single-shot flashover probability $p_{u^{*}}$, associated with the observed average first breakdown voltage $\bar{u}^{*}=432.04$ $\mathrm{kV}$. Inserting the sample standard deviation in voltage steps, $s^{*} / \Delta U=2.99$, into (39) we obtain

$$
\hat{p}_{u^{*}}=\frac{0.0072}{2.99}=0.0024
$$

which implies that the average first breakdown voltage $\bar{u}^{*}=432.04 \mathrm{kV}$ is an estimate of the $0.24 \%$ quantile in the flashover probability function. As a rough check of the calculations we might enter Table 1 with the normalized step size $\Delta_{1} U / \beta=0.43 / m \simeq 0.004$. The corresponding value $p_{u^{*}}$ is found to be $p_{u^{*}}=0.2 \%$, which is satisfactory.

Table 2.

Voltage levels of first breakdown in $n=10$ series of an extended inverse sampling procedure with $U_{\text {at }}=358 \mathrm{kV}, \Delta_{m} U=2.14 \mathrm{kV}$, and $m=100$ voltage applications per step.

\begin{tabular}{|c||c|c|c|c|c|}
\hline \hline Series No. $i$ & 1 & 2 & 3 & 4 & 5 \\
\hline $\bar{U}_{s t} \mathrm{kV}$ & 358 & 358 & 358 & 358 & 358 \\
\hline $\begin{array}{c}\text { First breakdown } \\
\text { voltage } u_{i} \mathrm{kV}\end{array}$ & 424.34 & 430.76 & 430.76 & 435.04 & 437.18 \\
\hline Step number $j_{i}$ & 31 & 34 & 34 & 36 & 37 \\
\hline Shot number & 23 & 35 & 37 & 77 & 35 \\
\hline \hline Series No. $i$ & 6 & 7 & 8 & 9 & 10 \\
\hline$U_{\text {st }} \mathrm{kV}$ & 358 & 358 & 358 & 358 & 358 \\
\hline $\begin{array}{c}\text { First breakdown } \\
\text { voltage } u_{i} \mathrm{kV}\end{array}$ & 432.90 & 439.32 & 426.48 & 422.20 & 441.46 \\
\hline Step number $j_{i}$ & 35 & 38 & 32 & 30 & 39 \\
\hline Shot number & 97 & 27 & 8 & 14 & 56 \\
\hline \hline
\end{tabular}




\section{ROBUSTNESS OF THE INVERSE SAMPLING PROCEDURE}

$T^{N}$ the previous Sections we have assumed that the flashLover probability function could be described by a double exponential distribution. This assumption is, however, not as restrictive as it might appear. It may be argued that the first-breakdown distribution given by (13) is the natural limiting distribution for results obtained under the inverse sampling procedure when the underlying flashover probability function has an exponentially decreasing lower tail.

To illustrate the robustness of the procedure we have determined the exact first-breakdown distribution (7) under various assumptions of the underlying flashover probability function. We have investigated a normal distribution and Weibull distributions with shape parameters 1 , 2 and 5 , respectively. Since the properties of the inverse sampling procedure essentially depend on the flashover probability function in the neighborhood of the estimated quantile, the distributions have been selected to have the same $0.1 \%$ quantile and the same slope of the probability function at this quantile. Figure 4 shows the single shot flashover probability in the neighborhood of the $0.1 \%$ quantile for these distributions, and Figure 5 illustrates the more global properties.

For each of these probability functions we determined the exact first-breakdown distribution (7) under the inverse sampling procedure, and under the extended inverse sampling procedure with $m=10,20,50$ and 100 , using normalized step sizes $\Delta_{m} U / \beta=1.0,0.5,0.2,0.1,0.05$, $0.02,0.01,0.005,0.002$, and 0.0002 , respectively. We determined the expected first breakdown voltage $E\left[u^{*}\right]$ given by (9), the corresponding single-shot flashover probability $p^{*}=F\left(E\left[u^{*}\right]\right)$, and the normalized standard deviation $\sqrt{V\left[u^{*}\right]} / \Delta_{m} U$ (in voltage steps) in the first-breakdown distribution.

The result is illustrated in Figure 6 . The Figure shows the relation between the estimated flashover probability $p^{*}=F\left(E\left[u^{*}\right]\right)$, and the standard deviation $\sqrt{V\left[u^{*}\right]} / \Delta_{m} U$ in the first-breakdown distribution. It is seen that the approximative relation (41) yields a good approximation also for these probability functions.

\section{CONCLUSION}

$T_{\text {HE inverse sampling procedure is well suited for deter- }}$ mination of low quantiles in situations with a limited

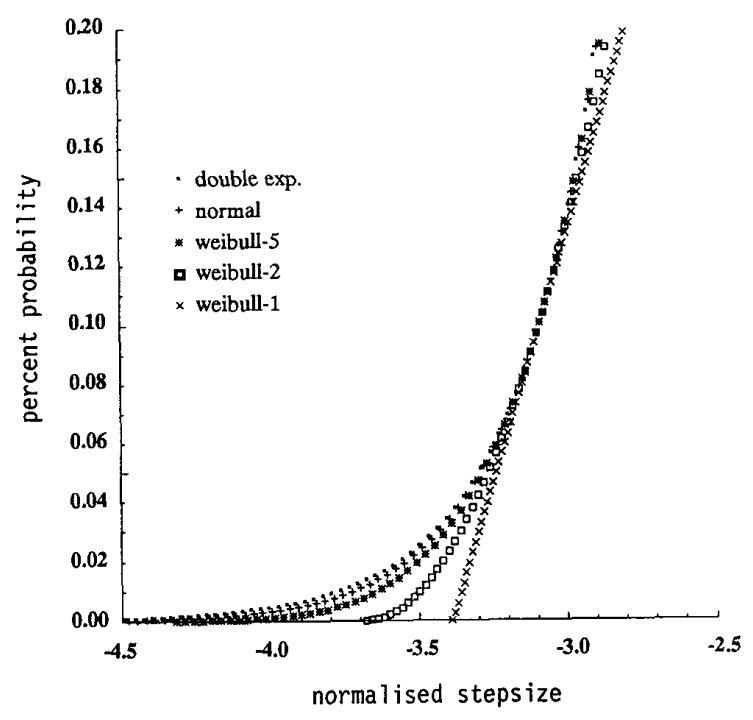

Figure 4.

Lower tail of flashover probability functions with matching 0.1 per cent quantile. The probability functions have the same slope at the 0.1 per cent quantile. The step size is normalized to the standard deviation of the normal distribution, with the normal distribution 50 per cent quantile taken as the origin.

number of test objects when the test object is damaged by a breakdown, but not affected by individual tests that do not result in breakdown.

\section{APPENDIX}

\subsection{THE APPROXIMATING FIRST-BREAKDOWN DISTRIBUTION FOR A DOUBLE EXPONENTIAL FLASHOVER PROBABILITY FUNCTION}

$\mathbf{W}^{\mathrm{E}}$ consider the first-breakdown distribution (7) in the case where the flashover probability function is given by a double exponential distribution with parameters $\alpha$ and $\beta$

$$
F(u)=1-\exp \left\{-\exp \left(\frac{u-\alpha}{\beta}\right)\right\}
$$

The probability that the first breakdown voltage ex- 


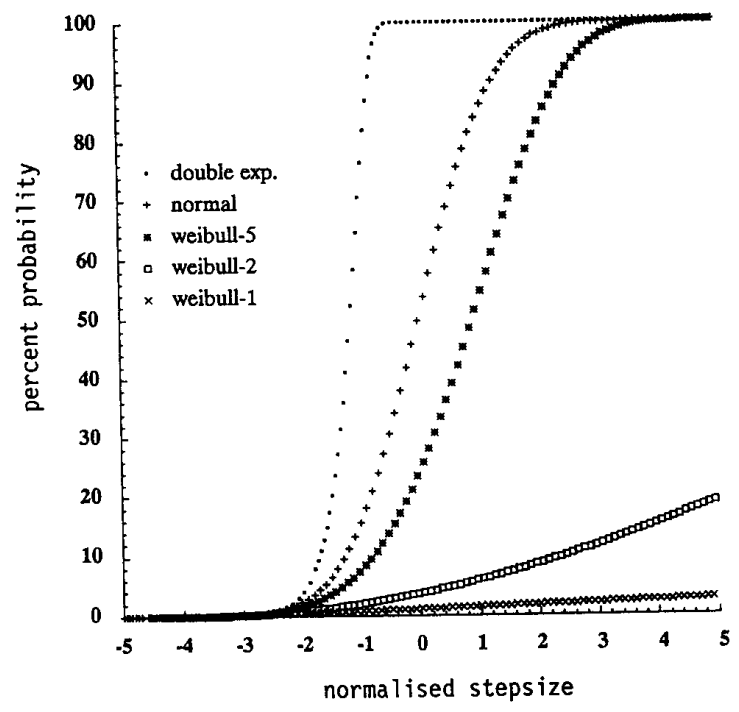

Figure 5.

Flashover probability functions with matching the $0.1 \%$ quantile. The probability functions have the same slope at the $0.1 \%$ quantile. The step size is normalized to the standard deviation of the normal distribution, with the normal distribution $50 \%$ quantile taken as the origin.

ceeds $u_{j}(j=1,2, \ldots)$ is

$$
\begin{aligned}
P[J>j] & =\left[\prod_{\nu=1}^{j}\left\{1-F\left(u_{\nu}\right)\right\}\right] \\
& =\exp \left\{-\sum_{\nu=1}^{j} \exp \left(\frac{U_{s t}+\nu \Delta U-\alpha}{\beta}\right)\right\} \\
j & =1,2, \ldots
\end{aligned}
$$

Utilizing that

$$
\begin{aligned}
\sum_{\nu=1}^{j} \exp \left(\frac{\nu \Delta U}{\beta}\right) & =\frac{\exp (\Delta U / \beta)}{\exp (\Delta U / \beta)-1}\{\exp (j \Delta U / \beta)-1\} \\
& =\exp (\eta)\{\exp (j \Delta U / \beta)-1\}
\end{aligned}
$$

with $\eta$ given by

$$
\eta=\log \left[\frac{\exp (\Delta U / \beta)}{\exp (\Delta U / \beta)-1}\right]
$$

we obtain

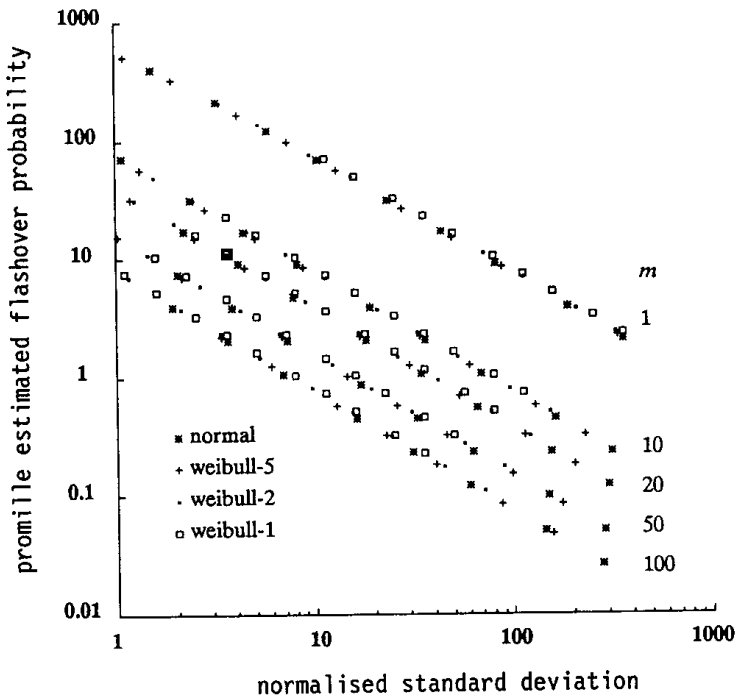

Figure 6.

The estimated flashover probability $p^{*}=F\left(E\left[u^{*}\right]\right)$ and the normalized standard deviation $\sqrt{V\left[u^{*}\right]} / \Delta_{m} U$ of the first breakdown voltage under the inverse sampling procedure with $m=1,10,20,50$ and 100 , assuming a normal, a Weibull-1, Weibull-2 and a Weibull-5 flashover probability function. The flashover probability functions are illustrated in Figures 4 and 5 . Normalized step sizes $\Delta_{m} U / \beta=$ $1.0 ; 0.5 ; 0.2 ; 0.1 ; 0.05 ; 0.02 ; 0.01 ; 0.005 ; 0.002$; and 0.0002 .

$$
\begin{aligned}
P[J>j] & =\exp \left\{-\exp \left(\frac{U_{s t}+\eta \beta-\alpha+j \Delta U}{\beta}\right)\right. \\
& \left.-\exp \left(\frac{U_{s t}+\eta \beta-\alpha}{\beta}\right)\right\} \\
& =\frac{1-F\left(u_{j}+\eta \beta\right)}{1-F\left(U_{s t}+\eta \beta\right)} \quad j=1,2, \ldots
\end{aligned}
$$

Since

$$
p_{j}=P[J>j-1]-P[J>j]
$$

we have that the first-breakdown probabilities $p_{j}$ are of the form

$$
p_{j}=\frac{F\left(U_{s t}+\eta \beta+j \Delta U\right)-F\left(U_{s t}+\eta \beta+(j-1) \Delta U\right)}{1-F\left(U_{s t}+\eta \beta\right)}
$$

for $\quad j=1,2, \ldots$

Introducing the continuous distribution $F^{*}$ as

$$
F^{*}(u)= \begin{cases}\frac{F(u+\eta \beta)}{F\left(U_{n t}+\eta \beta\right)} & \text { if } u>U_{s t} \\ 0 & \text { if } u \leqslant U_{s t}\end{cases}
$$


we find that $p_{j}$ may be expressed as

$$
p_{j}=F^{*}\left(u_{j}\right)-F^{*}\left(u_{j-1}\right) \quad j=1,2, \ldots
$$

which shows that the discrete distribution $p_{j}$ is determined as the probability associated with the interval $\left[u_{j-1}, u_{j}\right]$ in the continuous distribution $F^{*}$. Therefore, $F^{*}$ will be termed 'the approximating first-breakdown distribution'.

\section{REFERENCES}

[1] W. J. Dixon and A. M. Mood, "A Method for Obtaining and Analyzing Sensitivity Data", J. Amer. Statist. Assoc., Vol. 43, pp. 109-126, 1948.

[2] S. Vibholm and P. Thyregod, "A Study of the Up-and-Down Method for Non-normal Distribution Functions", IEEE Trans. Elect. Insul., Vol. 23, pp. 357-364, 1988.
[3] G. Carrera and L. Dellera, "Accuracy of an Extended Up and Down Method in Statistical Testing of Insulation", Electra, No. 23, pp. 159-175, 1972.

[4] M. S. Bartlett, "A Modified Probit Technique for Small Probabilities", Suppl. J. Roy. Statist. Soc., Vol. 8, pp. 113-117, 1946.

[5] S. Vibholm, A. Pedersen, J. M. Christensen and P. Thyregod, "The Effect of Surface Roughness on Low Probability First Breakdown in Compressed $\mathrm{SF}_{6}$ ", paper 32.06, Third International Symposium on $\mathrm{HV}$ Engineering, Milan 1979.

[6] D. McLeish and D. Tosh, "The Estimation of Extreme Quantiles in Logit Bioassay", Biometrika, Vol. 70, pp. 625-632, 1983.

Manuscript was received on 22 March 1991, in revised form 29 April 1991. 